\title{
CONSIDERAÇÕES DE INTERESSE AO CIRURGIÃO-DENTISTA NO ATENDIMENTO AO PACIENTE PORTADOR DE SARCOIDOSE
}

\section{CONSIDERATIONS OF INTEREST TO THE DENTIST IN THE MANAGEMENT OF THE PATIENT WHO IS A CARRIER OF SARCOIDOSIS}

\author{
Irineu Gregnanin Pedron', Sandra Regina Echeverria Pinho da Silva ${ }^{2}$, \\ Carlos Alberto Adde ${ }^{3}$, José Carlos Pettorossi Imparato ${ }^{4}$ \\ 1 Autor para contato: Rua Flores do Piauí, 347, São Paulo - SP; (11) 6944-4067; \\ e-mail: igpedron@apcd.org.br \\ 2 Pontifícia Universidade Católica - PUC, Campinas, SP, Brasil. \\ 3 Faculdade de Odontologia da USP - FOUSP, Departamento de Estomatologia, São \\ Paulo, SP, Brasil; e-mail: caadde@usp.br \\ 4 Programa de Pós-graduação em Odontopediatria da São Leopoldo Mandic, Campinas, \\ SP; Faculdade de Odontologia da USP e PUC-Campinas; e-mail: jimparato@uol.com.br
}

Recebido para publicação em 26/02/2004

Aceito para publicação em 01/09/2004

\section{RESUMO}

A sarcoidose é uma doença granulomatosa crônica, de etiologia desconhecida, que acomete vários sistemas, incluindo o sistema estomatognático. Na cavidade bucal são característicos os granulomas sarcóides na mucosa bucal, palato, língua, gengiva e nos ossos maxilares, sob forma de lesões radiolúcidas semelhantes às lesões císticas. Acomete normalmente as glândulas salivares maiores, provocando distúrbios deletérios como a xerostomia, elevando a freqüência de lesões cariosas, doenças periodontais, mucosite e candidíase. O propósito deste trabalho foi apresentar o caso clínico de uma paciente portadora de sarcoidose com algumas manifestações bucais. Foram abordadas as considerações de interesse ao cirurgião-dentista para o correto atendimento e cuidados eventuais ao paciente portador da sarcoidose. Foram discutidas as particularidades da doença, como as manifestações clínicas gerais e do sistema estomatognático, incidência e freqüência, etiopatogênese, diagnóstico diferencial e terapia sistêmica.

Palavras-chave: sarcoidose, manifestações bucais, cavidade bucal, lesões

\begin{abstract}
Sarcoidosis is a chronic granulomatous disease, of unknown etiology, that
\end{abstract}


involves many systems, including the stomatognathic system. In the oral cavity are characteristics the sarcoid granulomas in oral mucosa, palate, tongue, gingiva and, in the maxillary bones are common in the oral cavity in the form of radiolucent lesions similar to the cystic lesions. It happens normally at the salivary glands, provoking deleterious disturbances how the xerostomia, increasing the frequence of caries, periodontal diseases, mucositis and candidiasis. The purpose of this paper was to present the case of a female patient carrier of sarcoidosis with some oral manifestations. The considerations of interest to the dentist for the correct assistance and eventual care to the patient carrier of sarcoidosis were involved. The particularities of this disease were discussed, as the general and oral clinic manifestations, incidence and frequency, etiopathogenesis, differential diagnosis, systemic therapy and implications in the odontological assistance.

Key words: sarcoidosis, oral manifestations, mouth, injuries

\section{Introdução}

A sarcoidose é considerada uma doença sistêmica granulomatosa crônica, de etiologia desconhecida, caracterizada pela presença de granulomas não caseificantes, constituídos pelo acúmulo de linfócitos T e fagócitos mononucleares nos órgãos envolvidos. Afeta principalmente o sistema retículo-endotelial: nódulos linfáticos hilares, pulmão e fígado (Ammirati et al., 1986; Calderon et al., 1990; Steinberg e Mueller, 1994; Blinder et al., 1997; Piatelli et al., 1998; Surattanontet al., 2002). O curso clínico pode ser autolimitante e subclínico, evoluindo à resolução espontânea, ou progressivo e fulminante, requerendo longo tratamento com corticosteróides, variando de 6 a 8 meses (Deluke e Sciubba, 1985; Ammirati et al., 1986; Piatelli et al., 1998; Hong e Farish, 2000). Os sinais e sintomas incluem tosse seca, dispnéia, febre, letargia, fadiga, perda de peso, exantemas e mal estar (Deluke e Sciubba, 1985; Ammirati et al., 1986; Mandel e Kaynar, 1994; Steinberg e Mueller, 1994; Serrat Soto et al., 1997; Piatelli et al., 1998; Hong e Farish, 2000; Lindeboom e Van Den Akker, 2000; Surattanontet al., 2002). Aproximadamente 50\% dos casos apresentam envolvimento pulmonar, caracterizados pela linfadenopatia hilar bilateral, causando insuficiência pulmonar ecor pulmonale (Jaeger et al., 1983; Deluke e Sciubba, 1985; Ammiratiet al., 1986; Calderon et al., 1990; Mandel e Kaynar, 1994; Steinberg e Mueller, 1994; Blinder et al., 1997; Serrat
Soto et al., 1997; Piatelli et al., 1998; Batal et al., 1999; Hong e Farish, 2000; Lindeboom e Van Den Akker, 2000; Surattanontet al., 2002). A tuberculose foi considerada uma seqüela, de acordo com Deluke e Sciubba (1985) e Hong e Farish (2000). Entretanto, outros órgãos e sistemas podem ser afetados, variando a freqüência. Na pele são comuns placas violáceas endurecidas e infiltrativas, caracterizando o eritema nodoso. Quando estes estão presentes no nariz, bochecha, ouvido, testa e mãos, particularizam o lúpus pernio (Van Maarsseveen et al., 1982; Jaeger et al., 1983; Deluke e Sciubba, 1985; Ammirati et al., 1986; Mandel e Kaynar, 1994; Steinberg e Mueller, 1994; Serrat Soto et al., 1997; Piatelli et al., 1998; Batal et al., 1999; Hong e Farish, 2000; Lindeboom e Van Den Akker, 2000;). O envolvimento ocular é incomum, com inflamação do trato uveal anterior (uveíte), glaucoma secundário ou catarata (Van Maarsseveen et al., 1982; Jaeger et al., 1983; Deluke e Sciubba, 1985; Ammirati et al., 1986; Mandel e Kaynar, 1994; Steinberg e Mueller, 1994; Blinder et al., 1997; Serrat Soto et al., 1997; Piatelli et al., 1998; Batalet al., 1999; Hong e Farish, 2000; Lindeboom e Van Den Akker, 2000; Surattanont et al., 2002). Foram citados ainda os envolvimentos ósseos, cardíacos, renais, hepáticos, esplênicos, em glândulas salivares, laringe, trato gastrointestinal, neurais e em nariz e seios maxilares (Van Maarsseveen et al., 1982; Jaeger et al., 1983; Deluke e Sciubba, 1985; Ammirati et al., 1986; Calderon et al., 1990; Mandel e Kaynar, 1994; 
Steinberg e Mueller, 1994; Blinder et al., 1997; Serrat Soto et al., 1997; Piatelli et al., 1998; Batal et al., 1999; Hong e Farish, 2000; Lindeboom e Van Den
Akker, 2000; Surattanont et al., 2002). As alterações e manifestações clínicas foram sintetizadas no Quadro 1.

Quadro 1 - Órgãos e/ou sistemas envolvidos, e suas manifestações clínicas na sarcoidose sistêmica.

\begin{tabular}{|c|c|}
\hline Órgãos & Alterações e Manifestações Clínicas \\
\hline Pulmões & $\begin{array}{l}90 \% \text { dos pacientes têm achados radiográficos incomuns; achado acidental; } \\
\text { fibrose pulmonar decorrente; linfadenopatia hilar bilateral característica; } \\
\text { envolvimento do parênquima é variável. }\end{array}$ \\
\hline Pele & $\begin{array}{l}\text { Presente em 25\% dos pacientes; manifestações cutâneas, caracterizadas por } \\
\text { granulomas máculopapulares, lúpus pernio (lesões endurecidas, violáceas ou } \\
\text { eritematosas em nariz, bochechas, lábios, orelhas e joelhos) ou eritema nodoso } \\
\text { (nódulos eritematosos subcutâneos, macios à palpação); pode estar associada a } \\
\text { vitiligo adjacente. }\end{array}$ \\
\hline Olhos & $\begin{array}{l}\text { 25\% de envolvimento, sendo mais comum a inflamação do trato uveal anterior } \\
\text { (uveíte granulomatosa), com o aumento da glândula lacrimal e envolvimento do } \\
\text { nervo óptico, glaucoma secundário, embora possa ocorrer a ceratoconjuntivite } \\
\text { seca (olho seco, visão embaçada e dilacerante, fotofobia); as lesões não tratadas } \\
\text { podem evoluir para cegueira. }\end{array}$ \\
\hline Ossos & $\begin{array}{l}5 \% \text { na sarcoidose crônica. Lesões císticas radiolúcidas, em forma de "saca- } \\
\text { bocado", acometendo falanges, metacarpais e metatarsais; ossos do crânio, } \\
\text { nasais, costelas e vértebras podem ser afetados; poliartralgia; destruição das } \\
\text { paredes ósseas do seio e septo nasal; ossos maxilares e faciais, representados por } \\
\text { osteoporose, expansão da cortical, lesões císticas bem delimitadas, e destruição } \\
\text { óssea extensa. }\end{array}$ \\
\hline Coração & $\begin{array}{l}5 \text { a } 10 \% \text {, sendo características as disfunções (falha cardíaca congestiva, disritmia, } \\
\text { efusão pericardial recorrente, bloqueio cardíaco, angina pectoris); sarcoidose do } \\
\text { miocárdio conduz a arritmias paroxísticas e morte súbita. }\end{array}$ \\
\hline Rins & Nefrolitíase, nefrocalcinose com insuficiência renal. \\
\hline Baço & $\begin{array}{l}\text { Esplenomegalia em } 5 \text { a } 10 \% \text {. O hiperesplenismo pode causar a trombocitopenia, } \\
\text { leucopenia e anemia ocasionais. Hiperprodução de vitamina D com } \\
\text { hipercalciúria e hipercalcemia. }\end{array}$ \\
\hline Fígado & $\begin{array}{l}\text { O envolvimento hepático pode ser observado em } 40 \% \text { dos casos. A disfunção } \\
\text { hepática não é comum. Hepatite crônica e cirrose são raras. Tubérculos } \\
\text { incidentais, granulomas hepáticos e cirrose pós-necrótica com hipertensão portal } \\
\text { com elevação de bilirrubina, fosfatase alcalina e gama-glutamiltransferase. }\end{array}$ \\
\hline Glândulas salivares & $\begin{array}{l}\text { É freqüente o envolvimento bilateral das glândulas parótidas, caracterizado pelo } \\
\text { aumento de volume do parênquima (firme à palpação e assintomático), causando } \\
\text { a atrofia da glândula e eventual fibrose, originando a redução do fluxo salivar } \\
\text { (xerostomia). Pode haver regressão espontânea do aumento da massa glandular. }\end{array}$ \\
\hline Nervos & $\begin{array}{l}\text { Nervos cranianos, particularmente o } \mathrm{N} \text { facial e o N. alveolar inferior, causando } \\
\text { paralisias faciais. Quando a glândula pituitária é afetada, pode desenvolver } \\
\text { diabetes insipidus, associado com o envolvimento do córtex cerebral, ataques } \\
\text { convulsivos podem ocorrer. }\end{array}$ \\
\hline $\begin{array}{l}\text { Nariz e seios } \\
\text { maxilares }\end{array}$ & $\begin{array}{l}\text { O envolvimento sinusal é raro. } 1 \text { a } 6 \% \text { acomete nariz e seios maxilares. } \\
\text { Obstrução nasal, epistaxe, dor, descarga e crostas, epífora, anosmia, hiposmia, } \\
\text { dispnéia, hipertrofia polipóide, sinusite crônica e mucosa friável estão presentes } \\
\text { em } 20 \% \text { dos casos. Pode destruir paredes ósseas do seio e septo nasal. }\end{array}$ \\
\hline
\end{tabular}


A sarcoidose pode apresentar-se em dois estágios. Na primeira fase, aguda, inflamatória e prégranulomatosa, os linfócitos T são predominantes nos granulomas. Acomete indivíduos jovens de até 30 anos. As características clínicas são de início abrupto: poliartrite; linfadenopatia hilar bilateral; febre; uveíte; e eritemas nodosos, caracterizando a Síndrome de Löfgren. O prognóstico é bom, com resolução completa dos sintomas em 90\% dos casos (Ammirati et al., 1986; Serrat Soto et al., 1997; Batal et al., 1999; Hong e Farish, 2000). Nessa fase, a sarcoidose pode ser autolimitante, não necessitando de tratamento. A segunda fase, crônica e granulomatosa, é a forma ativa da doença. A resposta imune celular da sarcoidose parece ser ponteada por macrófagos, em resposta ao antígeno desconhecido. Os mediadores liberados por macrófagos, como a interleucina-1 (IL-1) estimulam o acúmulo e proliferação de linfócitos Thelper. Esses liberam mediadores (interferon-gama e linfocinas) que amplificam a resposta imune pelo recrutamento e ativação de fagócitos mononucleares, estabelecendo blocos para a formação do granuloma (Van Maarsseveen et al., 1982; Ammirati et al., 1986; Batal et al., 1999). Esses podem estar presentes em qualquer órgão ou sistema. Acomete indivíduos com mais de 40 anos. Progride ao envolvimento de vários sistemas, estando a mortalidade relacionada à fibrose pulmonar e insuficiência renal. Na fase crônica, corticosteróide para controlar a inflamação granulomatosa dos órgãos

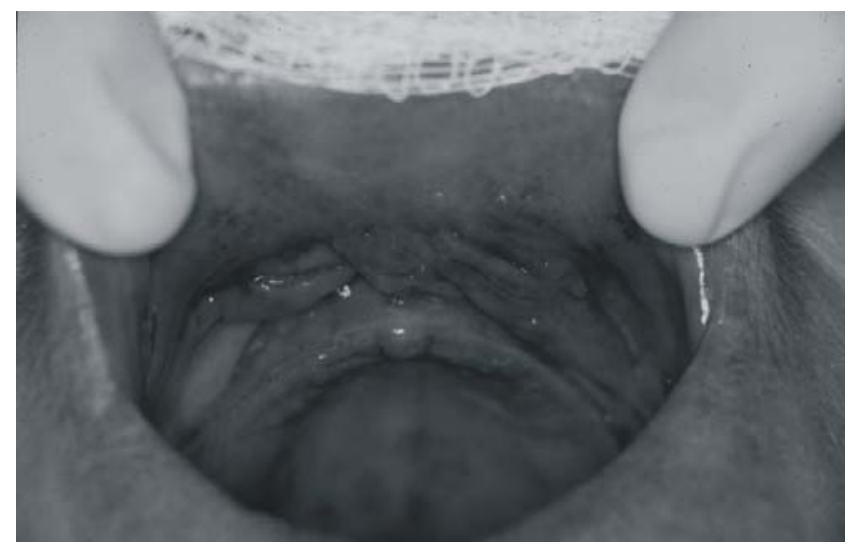

Figura 1 - Hiperplasia fibrosa inflamatória sob prótese total, na paciente portadora de sarcoidose. envolvidos é imprescindível. Quando o corticosteróide não resolve, a radioterapia pode ser indicada (Ammirati et al., 1986; Calderon et al., 1990; Batal et al., 1999).

O propósito deste trabalho foi apresentar o caso clínico de uma paciente portadora de sarcoidose com algumas manifestações bucais e as considerações de interesse ao cirurgião-dentista para o correto atendimento e cuidados eventuais.

\section{Relato de Caso Clínico}

Paciente do sexo feminino, leucoderma, 48 anos de idade, compareceu à clínica particular queixandose de desconforto sob a prótese. Em referência a seu histórico clínico, a paciente reportou ser portadora de sarcoidose, estando sob tratamento com corticosteróides endovenoso (prednisolona).

Clinicamente, a paciente apresentou prótese total superior mal adaptada, com lesão fibrosa, de coloração normal, localizada na região de fórnix dos dentes anteriores, indicativa de hiperplasia fibrosa inflamatória (Figura 1). Foi observada ainda a despapilação lingual, associada à xerostomia (Figura 2) e escoriações na região peribucal e rágade em vermelhão do lábio inferior (Figura 3). Ressecamento, exantemas e escoriações na região perinasal (Figura 4) e na região auricular (Figura 5) também foram observadas.

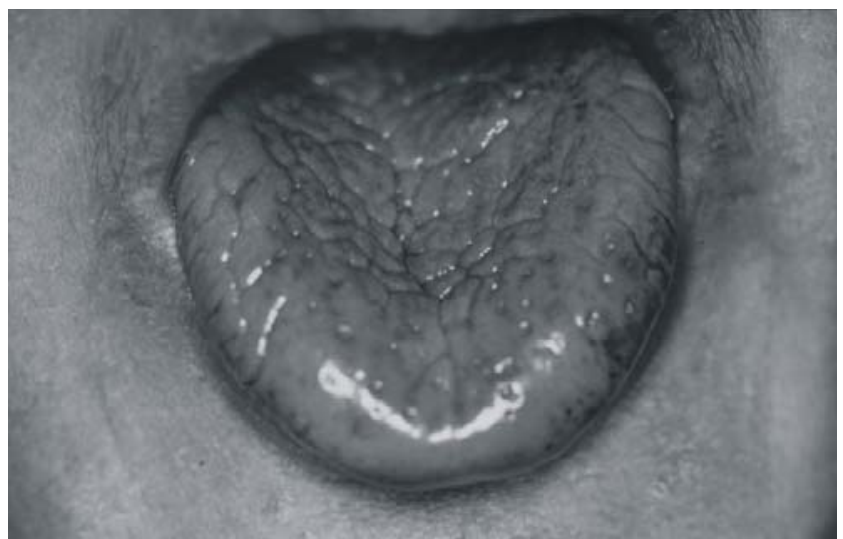

Figura 2 - Xerostomia em paciente portadora de sarcoidose, associada a despapilação lingual. 


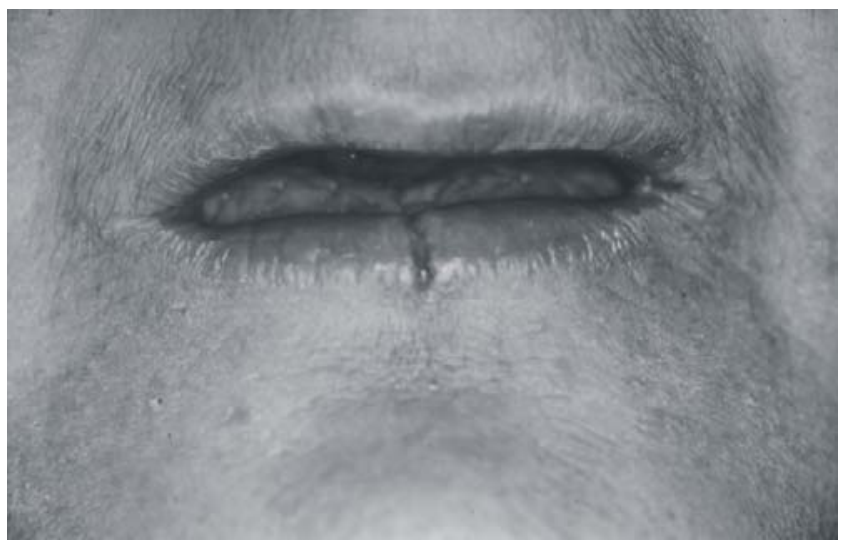

Figura 3 - Escoriações na região peribucal e rágade em vermelhão do lábio inferior, decorrentes da xerostomia.

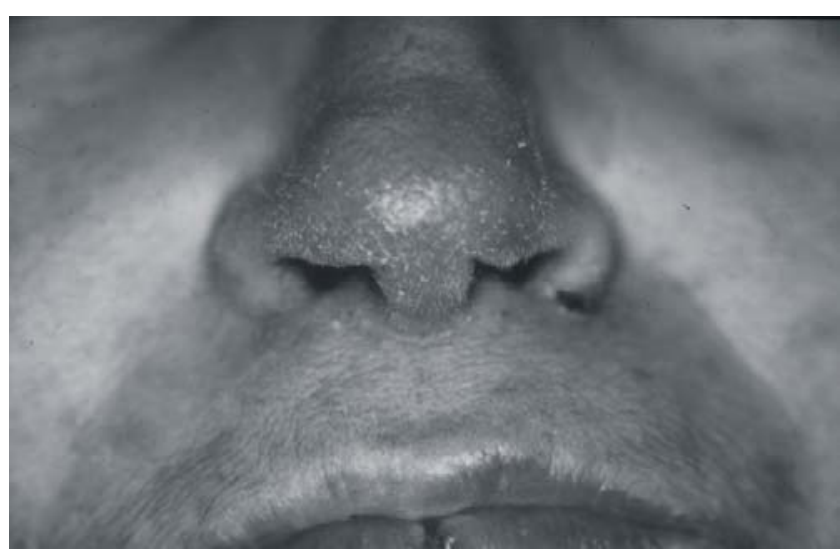

Figura 4 - Ressecamento, exantemas e escoriações na região perinasal.

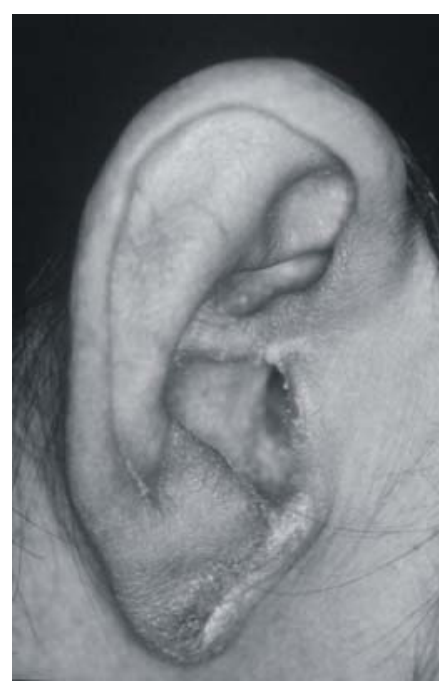

Figura 5- Ressecamento da pele e exantemas na região auricular.
O médico responsável foi contatado, sendo solicitada a avaliação do estado de saúde geral da paciente. Foi realizada biópsia incisional da lesão, sob anestesia local infiltrativa (mepivacaína a 3\%, sem vasoconstritor) e sob antibiótico profilático (protocolo American Heart Association), fixada em formol a $10 \%$ e encaminhada ao Laboratório de Patologia Cirúrgica do Departamento de Estomatologia da Faculdade de Odontologia da Universidade de São Paulo. O diagnóstico histopatológico foi hiperplasia fibrosa inflamatória.

Foi recomendada a utilização de bochechos com saliva artificial, sempre que houvesse sintomatologia decorrente da xerostomia e, confeccionada a nova prótese, foi mantida a hiperplasia fibrosa, pois sua presença auxiliou na estabilidade da prótese, em decorrência da pequena dimensão vertical do rebordo maxilar. Não foi possível a tomada da última fotografia clínica, pois a paciente mudou de endereço (cidade).

\section{Discussão}

A sarcoidose bucal, em glândulas salivares ou nódulos linfáticos da região cérvico-facial são infreqüentes, embora possam ocorrer na língua, mucosa bucal, palato duro e mole, gengiva, soalho e lábios (Deluke e Sciubba, 1985; Ammirati et al., 1986; Serrat Soto et al., 1997; Piatelli et al., 1998; Hong e Farish, 2000; Lindeboom e Van Den Akker, 2000). O padrão clínico normalmente é representado por nódulos violáceos ou azuis bem delimitados, não macios, podendo ter ocasional ulceração (Serrat Soto et al., 1997). Entretanto, segundo Blinder et al. (1997) e Batal et al. (1999), as características clínicas dependem da localização. Assim, o aumento de volume assintomático, irregular, submucoso, aderido ao tecido adjacente, mas “móvel” à palpação é comum na mucosa, embora possa ainda apresentar-se encapsulado. Na língua, o aumento de volume firme, endurecido e submucoso é observado, bem como lesões papulares erosivas. No soalho, é comum a característica de bolha, semelhante à rânula. Na gengiva, o aumento eritematoso com padrão nodular associado a ulcerações gengivais. No palato, múltiplos nódulos. No osso maxilar, é mais comum na região da pré- 
maxila, sendo características as lesões radiolúcidas difusas com bordas mal definidas até o soalho nasal. Nos dentes afetados pelas lesões, são achados comuns a mobilidade dentária progressiva e vitalidade sem reabsorção. Na mandíbula, as regiões de pré-molares e côndilos são mais afetadas. A xerostomia é freqüente e decorrente da redução do fluxo salivar quando do envolvimento das glândulas salivares, usualmente as parótidas, como observado no presente relato (Ammirati et al., 1986; Calderon et al., 1990; Mandel e Kaynar, 1994; Steinberg e Mueller, 1994; Batalet al., 1999; Surattanont et al., 2002). O aumento de volume da glândula parótida, com envolvimento histológico das glândulas salivares menores é o padrão clínico mais comum, sendo esperada a regressão espontânea. O segundo padrão clínico é a ausência de aumento da glândula salivar, embora haja a presença de granulomas não caseificantes, observados microscopicamente (Mandel e Kaynar, 1994; Surattanontet al., 2002). O terceiro padrão é a Síndrome de Heerfordt ou febre uveoparotídea, composta pela tríade de sintomas - aumento das glândulas salivares, uveíte e envolvimento dos nervos cranianos, sendo comuns as paralisias faciais e do lábio inferior (Van Maarsseveen et al., 1982; Jaeger et al., 1983; Deluke e Sciubba, 1985; Ammirati et al., 1986; Mandel e Kaynar, 1994; Serrat Soto et al., 1997; Hong e Farish, 2000; Surattanont et al., 2002).

A sarcoidose apresentou incidência levemente superior em mulheres do que em homens, numa proporção de 1,5:1, respectivamente. Acomete a faixa etária de 20 a 40 anos. Negros são mais afetados que brancos, numa proporção de 10 a 17:1 (Van Maarsseveen et al., 1982; Jaeger et al., 1983; Deluke e Sciubba, 1985; Ammirati et al., 1986; Steinberg e Mueller, 1994; Blinder et al., 1997; Serrat Soto et al., 1997; Piatelli et al., 1998; Hong e Farish, 2000; Lindeboom e Van Den Akker, 2000; Surattanont et al., 2002). Apresenta freqüência de 1 a 40 casos a cada 100.000 pessoas por ano (Ammiratiet al., 1986; Piatelli et al., 1998).

A etiopatogenia é desconhecida, havendo, entretanto, teorias, como a infecciosa e a imunológica (Jaeger et al., 1983; Deluke e Sciubba, 1985; Ammiratiet al., 1986; Mandel e Kaynar, 1994; Serrat Soto et al., 1997; Piatelli et al., 1998; Hong e Farish, 2000; Surattanont et al., 2002). Deluke e Sciubba
(1985) e Piatelli et al. (1998) sugeriram a depressão na imunidade celular mediada, manifestada por redução de linfócitos $\mathrm{T}$ circulantes e agentes infecciosos micobacterianos (Mycobacterium tuberculosis) ou vírus (HHV8) associados à predisposição genética. A transmissão corpo-a-corpo; exposição ao agente externo; transplante cardíaco e de medula óssea foram relacionados (Piatelliet al., 1998).

Não há testes específicos para o diagnóstico da sarcoidose, e a elucidação ocorre por exclusão (Ammirati et al., 1986; Lindeboom e Van Den Akker, 2000; Surattanont et al., 2002). Normalmente, os achados clínicos e radiográficos e a história médica são apoiados por evidências histopatológicas de granulomas epitelióides não caseificantes com exclusão de outras hipóteses diagnósticas (Ammirati et al., 1986; Steinberg e Mueller, 1994; Batal et al., 1999). A radiografia de tórax, a presença de anergina à tuberculina, elevada SACE (do inglês Serum Angiotensin Converting Enzyme ou enzima conversora de angiotensina sorológica), e resposta positiva do teste de Kveim, são usualmente empregados (Van Maarsseveen et al., 1982; Deluke e Sciubba, 1985; Ammirati et al., 1986; Calderonet al., 1990; Steinberg e Mueller, 1994; Blinder et al., 1997; Serrat Soto et al., 1997; Piatelliet al., 1998; Batal et al., 1999; Hong e Farish, 2000; Lindeboom e Van Den Akker, 2000; Surattanont et al., 2002). O teste de Kveim positivo é angariado com a injeção de suspensão estéril de tecido sarcóide humano sub-cutâneo. Lesões nodulares com tubérculos epitelióides na biópsia (de 4 a 6 semanas após a injeção) são observadas, em 50 a $80 \%$ dos pacientes portadores de sarcoidose (Jaeger et al., 1983; Deluke e Sciubba, 1985), e embora seja empregado, não foi aprovado pela FDA, assim como o SACE (Steinberg e Mueller, 1994; Piatelliet al., 1998; Batal et al., 1999). Culturas e pesquisas fúngicas e bacterianas devem ser negativas, como descarte de outras hipóteses diagnósticas (Deluke e Sciubba, 1985; Ammirati et al., 1986; Steinberg e Mueller, 1994; Surattanont et al., 2002). Os exames hematológicos não são diagnósticos, mas podem auxiliar no monitoramento da fase ativa da doença. Sedimentação de eritrócitos levemente aumentados; leucopenia; eosinofilia; anemia; trombocitopenia; hiperglobulinemia; aumento da absorção intestinal de cálcio, provocando hipercalcemia e hipercalciúria podem ser observados 
(Deluke e Sciubba, 1985; Ammirati et al., 1986; Calderon et al., 1990; Mandel e Kaynar, 1994; Steinberg e Mueller, 1994; Batal et al., 1999). Outros exames foram citados, como a lavagem broncoalveolar, caracterizada pelo aumento quantitativo de linfócitos Thelper; e a utilização do Gallium 67, que demonstra a atividade da doença (Ammirati et al., 1986; Steinberg e Mueller, 1994; Batal et al., 1999; Hong e Farish, 2000). Nenhum desses resultados e exames é patognomônico da sarcoidose (Calderonet al., 1990).

Dentre as características histopatológicas, que também não são patognomônicas, há pequenos granulomas compostos por histiócitos epitelióides, células gigantes de Langerhans, linfócitos e fibroblastos ocasionais. Ausência de necrose foi observada. As células gigantes podem conter ou não inclusões citoplasmáticas. Podem ainda ser encontradas no interior das células gigantes, inclusões estreladas como corpos asteróides e concreções laminadas compostas por cálcio e proteínas, denominadas de corpos de Schwmann (Van Maarsseveen et al., 1982; Deluke e Sciubba, 1985; Ammirati et al., 1986; Steinberg e Mueller, 1994; Piatelli et al., 1998; Surattanont et al., 2002).

Dependendo da localização das lesões e características clínicas apresentadas, um amplo diagnóstico diferencial clínico pode ser considerado na cavidade bucal. Assim, a sarcoidose nos maxilares imita granulomas eosinofílicos ou periodontite com lesão localizada no osso alveolar. Na gengiva podem ser admitidas a hiperplasia gengival medicamentosa e a gengivite de células plasmáticas; no soalho bucal, a rânula; na língua, palato, lábios e mucosa bucal, abscessos e tumores (Steinberg e Mueller, 1994; Blinder et al., 1997; Piatelli et al., 1998; Hong e Farish, 2000; Lindeboom e Van Den Akker, 2000). Quando do comprometimento de glândulas salivares, o aumento da massa do parênquima assemelha-se ao das lesões da Síndrome de Sjögren, diabetes melitus, má nutrição e etilismo (Hong e Farish, 2000). Tumores mesenquimais de glândulas salivares; hiperplasia focal de glândulas salivares menores; grânulos de Fordyce; fibroma; mucocele; schwanoma; neurofibroma; lesões granulomatosas da tuberculose; granulomas de corpos estranhos; sífilis; infecções fúngicas; lúpus eritematoso; doenças do colágeno (como artrite reumatóide e Síndrome de Sjögren) foram reportados (Ammirati et al., 1986; Calderon et al.,
1990; Steinberg e Mueller, 1994; Serrat Soto et al., 1997; Surattanont et al., 2002). Inerente ao diagnóstico diferencial histopatológico, o granuloma não caseificante pode apresentar-se em outras infecções micobacterianas (como a actinomicose) e fúngicas (blastomicose, toxoplasmose e histoplasmose), tuberculose, granulomatose orofacial, lepra, sífilis, doença de Crohn, Síndrome de Melkersson, doença de Hodgkin, e carcinoma (Jaeger et al., 1983; Deluke e Sciubba, 1985; Serrat Soto et al., 1997; Piatelliet al., 1998; Hong e Farish, 2000; Surattanont et al., 2002).

O tratamento normalmente é feito por meio de corticosteróides, para controlar a inflamação granulomatosa dos órgãos envolvidos, prevenindo a progressão da doença, embora não reverta as alterações fibróticas e localizadas no pulmão (Deluke e Sciubba, 1985; Ammirati et al., 1986; Calderon et al., 1990; Mandel e Kaynar, 1994; Steinberg e Mueller, 1994; Serrat Soto et al., 1997; Batal et al., 1999; Hong e Farish, 2000; Lindeboom e Van Den Akker, 2000; Surattanont et al., 2002). Segundo Batal et al. (1999), o corticosteróide pode controlar a regulação de vários mediadores envolvidos na patogênese da sarcoidose (interleucinas $1 \mathrm{e} 2$, interferon-gama e fator de necrose tumoral). O protocolo atual preconiza 30 a $40 \mathrm{mg}$ diários de prednisolona, de 6 a 12 semanas. Este protocolo é repetido nas crises agudas. Corticosteróides tópicos também devem ser administrados associados aos sistêmicos, em uso dermatológico ou oftalmológico (Batal et al., 1999; Surattanont et al., 2002). Cloroquina também foi associada ao corticosteróide (Hong e Farish, 2000; Lindeboom e Van Den Akker, 2000). Em doenças refratárias ou crônicas, drogas citotóxicas como metrotexato, azatioprina, clorambucil e ciclofosfamida foram empregados (Batal et al., 1999; Surattanont et al., 2002). Antibióticos para infecções secundárias e oportunistas também são administrados (Batal et al., 1999). Radioterapia foi citada, quando o corticosteróide não apresentou eficácia (Calderon et al., 1990; Batal et al., 1999; Lindeboom e Van Den Akker, 2000). Blinder et al. (1997) reportaram melhora significante com a utilização do ácido acetil salicílico na fase aguda, enquanto Deluke e Sciubba (1985) constataram que a utilização de agentes antimaláricos também apresentaram ação satisfatória. Excisão cirúrgica pode ser empregada em 
lesões bucais (Ammirati et al., 1986; Blinder et al., 1997; Serrat Soto et al., 1997). O laser $\mathrm{CO}_{2}$ foi recomendado na excisão de lúpus pernio associado às injeções de corticosteróide, apresentando excelentes resultados (Batalet al., 1999).

A relação entre sarcoidose e linfoma foi citada por Mandel e Kaynar (1994) e Piatelli et al. (1998). Segundo Mandel e Kaynar (1994), o aumento da atividade mitótica dos linfócitos causa o aumento do risco de mutação celular. Indivíduos portadores de sarcoidose têm 5,5 mais chances de desenvolver linfomas do que indivíduos normais. O linfoma de Hodgkin é comum em indivíduos mais idosos portadores de sarcoidose. Daí, a importância do cirurgião-dentista no aspecto preventivo na detecção inicial de linfomas na cavidade bucal.

O cirurgião-dentista desempenha papel fundamental na equipe multidisciplinar, já que as lesões bucais podem ser o primeiro sinal da sarcoidose, tornando a biópsia em tecidos bucais, necessária para auxiliar no diagnóstico da doença (Van Maarsseveen et al., 1982; Calderon et al., 1990; Serrat Soto et al., 1997; Hong e Farish, 2000; Surattanont et al., 2002).

Quanto ao tratamento odontológico, pacientes portadores de sarcoidose e sob ação de corticosteróide, devem ser avaliados pela insuficiência adrenal induzida pelo antiinflamatório corticosteróide. Normalmente, esses pacientes têm comprometimento de cicatrização e reparação, e susceptibilidade aumentada às infecções oportunistas. Usualmente se faz necessária antibioticoterapia profilática para procedimentos invasivos, como reportado no presente relato. A xerostomia é observada em pacientes com envolvimento de glândulas salivares e sob terapia com drogas citotóxicas. Essas drogas normalmente causam toxicidade gastrintestinal (provocando náuseas e vômitos) e hematológica (promovendo anemia e leucopenia), necessitando de monitoração. A interação entre odontólogo e médico é condiçãosine qua non. Mucosite, doenças periodontais, lesões cariosas e candidíase são decorrentes da xerostomia, e tratamentos preventivos com flúor tópico, antifúngicos, sialogogos e colinérgicos (pilocarpina) podem ser instituídos (Calderon et al., 1990; Batal et al., 1999).

De acordo com Calderon et al. (1990), a sarcoidose, acometendo as glândulas salivares pode ainda provocar eventual fibrose, promovendo adenopatia cervical, disfunções têmporo-mandibulares, destruição do osso maxilar e, após exodontias e cirurgias bucais, a reparação anormal dos sítios cirúrgicos.

\section{Conclusões}

É lícito concluir que a sarcoidose é uma doença granulomatosa insidiosa, de etiologia desconhecida, que atinge vários sistemas e órgãos, podendo acometer o sistema estomatognático. O curso assintomático e silencioso da doença dificulta a instituição do diagnóstico. Dessa forma o cirurgião-dentista desempenha um papel singular na equipe multidisciplinar, no diagnóstico ou suspeita inicial, já que as lesões bucais podem ser a primeira manifestação da doença. Nesses casos, a realização da biópsia é imprescindível. Pela possível existência da relação entre sarcoidose e linfoma, o caráter preventivo do cirurgião-dentista perante a oncologia também é definido. O diagnóstico inicial e tratamento rápido podem reduzir ou cessar a progressão da doença e destruição dos órgãos afetados. Considerado um paciente cujas necessidades são especiais, o portador da sarcoidose deve ser submetido a cuidados durante o tratamento odontológico, como a instituição de antibiótico profilático em procedimentos invasivos. Condutas e procedimentos conservadores devem ser eleitos como de primeira escolha, evitando expor o paciente às infecções e procedimentos invasivos.

\section{REFERÊNCIAS}

1 AMMIRATI, G.; SERPICO, R.; LAINO, G. Sarcoidosi e sue manifestazioni orali. Arch Stomatol, Napoli, v. 27, n. 2, p. 213234, 1986.

2 BATAL, H.; LEE CHOU, L.; COTTRELL, D.A. Sarcoidosis: medical and dental implications. Oral Surg Oral Med Oral Pathol Oral Radiol Endod, St. Louis, v. 88, n. 4, p. 386-390, 1999.

3 BLINDER, D.; YAHATOM, R.; TAICHER, S. Oral manifestations of sarcoidosis. Oral Surg Oral Med Oral Pathol Oral Radiol Endod, St. Louis, v. 83, n. 4, p. 458-461, 1997. 
4 CALDERON, S.; ANAVI, Y.; MAZAR, A.; BEN-BASSAT, M. Sarcoidosis with oral involvement. Ann Dent, Albany, v. 49, n. 1, p. 21-24, 1990.

5 DELUKE, D.M.; SCIUBBA, J.J. Oral manifestations of sarcoidosis: report of a case masquerading as a neoplasm. Oral Surg Oral Med Oral Pathol, St. Louis, v. 59, n. 2, p. 184188, 1985.

6 HONG, J.; FARISH, S.E. Intraosseus sarcoidosis of the maxilla: case report. J Oral Maxillofac Surg, Philadelphia, v. 58, n. 4, p. 435-439, 2000.

7 JAEGER, R.G; ARAÚJO, V.C.; ARAÚJO, N.S.; MARCUCCI, G.; PIRATININGA, J.L.; KARULIS, O.M.S. Sarcoidose bucal. Rev Assoc Paul Cirurg Dent, São Paulo, v. 37, n. 3, p. 210-221, 1983.

8 LINDEBOOM, J.A.H.; VAN DEN AKKER, H.P. Sinusitis as the first indication of sarcoidosis. An incidental finding in a patient with presumed "odontogenic" sinusitis: case report. Br J Oral Maxillofac Surg, London, v. 38, n. 4, p. 277-279, 2000.

9 MANDEL, L.; KAYNAR, A. Sialadenopathy: a clinical herald of sarcoidosis: report of two cases. J Oral Maxillofac Surg, Philadelphia, v. 52, n. 11, p. 1208-1210, 1994.

10 PIATELLI, A.; FAVIA, G.F.; DI ALBERTI, L. Oral ulceration as a presenting sign of unknown sarcoidosis mimicking a tumour: report of 2 cases. Oral Oncol, Oxford, v. 34, p. 427430, 1998.

11 SERRAT SOTO, A.; VALENTÍN, P.L.; REDONDO GONZÁLEZ, L.M.; SANZ SANTA CRUZ, C.; VERRIER HERNÁNDEZ, A. Oral sarcoidosis with tongue involvement. Oral Surg Oral Med Oral Pathol Oral Radiol Endod, St. Louis, v. 83, n. 6, p. 668-671, 1997.

12 STEINBERG, M.J.; MUELLER, D.P. Treating oral sarcoidosis. J Am Dent Assoc, Chicago, v. 125, n. 1, p. 76-79, 1994.

13SURATTANONT, F.; MANDEL, L.; WOLINSKY, B. Bilateral parotid swelling caused by sarcoidosis. J Am Dent Assoc, Chicago, v. 133, n. 6, p. 738-741, 2002.

14 VAN MAARSSEVEEN, A.C.M.T.; VAN DER WAAL, I.; STAM, J.; VELDHUIZEN, R.W.; VAN DER KWAST, W.A.M. Oral involvement in sarcoidosis. Int J Oral Surg, Copenhagen, v. 11, n. 1, p. 21-29, 1982. 